Fluctuation and Noise Letters

Vol. 0, No. 0 (2001) 000-000

(C) World Scientific Publishing Company

\title{
DYNAMICS IMPORTANCE SAMPLING FOR THE COLLECTION OF SWITCHING EVENTS IN VERTICAL-CAVITY SURFACE-EMITTING LASERS
}

\author{
STEFANO BERI, PETER V. E. McCLINTOCK \\ Physics Department, Lancaster University, Lancaster LA1 4YB, UK \\ RICCARDO MANNELLA \\ Dipartimento di Fisica, Università di Pisa and INFM UdR Pisa, Via Buonarroti 2, 56100 Pisa, \\ Italy \\ Received (received date) \\ Revised (revised date) \\ Accepted (accepted date)
}

\begin{abstract}
A numerical approach based on dynamic importance sampling (DIMS) is applied to investigate polarization switches in vertical-cavity surface-emitting lasers. A polarization switch is described as an activation process in a two-dimensional nonequilibrium system. DIMS accelerates the simulations and allows access to noise intensities that were previously forbidden, revealing qualitative changes in the shape of the transition paths with noise intensity.

Keywords: Activation processes, Nonequilibrium Systems, Numerical Simulations, Optimal trajectories, VCSEL
\end{abstract}

\section{Introduction}

Activation processes form a very wide and important subject within modern physics. Due to the presence of noise in real systems, dramatic qualitative changes in the dynamics may be observed [1-4]. The strong changes in the polarization-resolved light intensity in vertical cavity surface emitting lasers (VCSELs) [5] represent a particularly remarkable example of activation processes. VCSELs are a relatively new class of semiconductor lasers [6,7]. Among the many features that make the VCSEL a promising device for applications, one of the most important is its emission of a narrow beam that is more nearly circular than those from traditional edge emitting devices. This is due to the cylindrical design of the active cavity, and it makes VCSELs more effective in driving energy into optical fibres than standard lasers. However, applications requiring a well defined polarization state are limited by a spontaneous switching of the device between two orthogonal linearly polarized modes [8]. 
The use of numerical simulation to investigate such switching processes is limited by the exponentially long times required to collect a statistically satisfactory distribution of events. Different methods have been proposed to speed up the simulations. For example in [9] the process of building the probability distribution of a nonequilibrium system is accelerated by performing the simulations by steps and then matching two consecutive "slices" of the distribution. However, this method is not effective when activation events are considered. In [10] a scheme based on dynamic importance sampling (DIMS) was proposed, allowing fast simulations of one-dimensional dynamical systems in the limit of very small noise intensity. In [11] the method was extended to systems of higher dimensionality. So far, however it has been tested successfully only on simple models such as the overdamped driven Duffing and inverted van der Pol oscillators, and it has not yet been applied to systems of practical interest such as VCSELs.

The DIMS algorithm accelerates the simulations in the following way. Instead of exploring irrelevant parts of the coordinate-space, the simulations are performed in the vicinity of the trajectory that the laser is most likely to follow during a switch. Such a most probable escape path (MPEP) can be obtained by solution of an auxiliary Hamiltonian system $[12,13]$.

In this letter, we apply the DIMS scheme to the dynamics of VCSEL polarization. The most probable escape path is calculated theoretically using the Hamiltonian technique, and the numerical simulations are performed for a wide range of noise intensities. The transition from the basin of attraction of one linearly polarized mode to the orthogonally polarized one is shown to alter its path with noise intensity. Some of the results discussed here were presented during SPIE Second International Symposium on Fluctuations and Noise [14].

\section{Theory}

Consider a device with small linear dichroism emitting in the transverse fundamental mode [5] whose polarization may switch between two orthogonal directions due to spontaneous emission.

When the dichroism is small enough (i.e. when pumping is sufficiently large), the changes in the beam intensity during the polarization dynamics can be neglected. Under this condition, the polarization dynamics can be described using the polarization angle $\phi$ and the ellipticity $\chi$ :

$$
\begin{aligned}
\dot{\chi} & =f_{\chi}(\chi, \phi)+\xi_{\chi}(t) \\
f_{\chi} & =\omega_{l} \sin (2 \phi)-\gamma_{n} \sin (2 \chi) \cos (2 \chi)+\gamma_{l} \sin (2 \chi) \cos (2 \phi)+\xi_{\chi}(t) \\
\dot{\phi} & =f_{\phi}(\chi, \phi)+\xi_{\phi}(t) \\
f_{\phi} & =-\omega_{l} \frac{\sin (2 \chi)}{\cos (2 \chi)} \cos (2 \phi)+\gamma_{l} \frac{\sin (2 \phi)}{\cos (2 \chi)}-\omega_{n} \sin (2 \chi)+\xi_{\phi}(t),
\end{aligned}
$$

$\chi$ varies between $-\frac{\pi}{4}$ and $\frac{\pi}{4}$ and $\phi$ between 0 and $\pi$. With a proper choice of the parameters, two linear polarization modes are stable: the $\hat{x}$-polarized mode (corresponding to $\chi=0$ and $\phi=0$ ) and the $\hat{y}$-polarized mode (corresponding to $\chi=0$ and $\phi=\frac{\pi}{2}$ ). The basins of attractions of the two polarization modes are separated by an unstable limit cycle. The parameters $\omega_{l}$ and $\gamma_{l}$ are respectively the 
linear birefringence and linear dichroism; $\gamma_{n}$ and $\omega_{n}$ are the nonlinear anisotropies. The stochastic terms $x i_{\chi, \phi}$ describe the spontaneous emission. They are additive in Eq.(1)-(3). However, their intensity is related to the beam intensity and their effect is ultimately multiplicative. Yet, for a device with small dichroism and operating with large enough pumping, the intensity can be considered to be a constant:

$$
\begin{aligned}
\left\langle\xi_{\chi}(t)\right\rangle & =\left\langle\xi_{\phi}(t)\right\rangle=0 \quad \forall t \\
\left\langle\xi_{\chi}(t) \xi_{\chi}(s)\right\rangle & =\left\langle\xi_{\phi}(t) \xi_{\phi}(s)\right\rangle=\epsilon \delta(t-s) \quad\left\langle\xi_{\phi}(t) \xi_{\chi}(s)\right\rangle=0 \quad \forall t, s
\end{aligned}
$$

The time evolution of the probability density for the system (2), (4), is regulated by the following Fokker-Planck equation

$$
\frac{\partial \varrho}{\partial t}=-\frac{\partial\left(f_{\chi} \varrho\right)}{\partial \chi}-\frac{\partial\left(f_{\phi} \varrho\right)}{\partial \phi}+\frac{\epsilon}{2}\left(\frac{\partial^{2} \varrho}{\partial \chi^{2}}+\frac{\partial^{2} \varrho}{\partial \phi^{2}}\right)
$$

In the limit $\epsilon \rightarrow 0,(7)$ can be solved using the WKB expansion $[12,13]$. The probability distribution is considered to have the form

$$
\varrho(\chi, \phi, t)=z(\chi, \phi, t) \exp \left(-\frac{S(\chi, \phi, t)}{\epsilon}\right), \quad \epsilon \rightarrow 0,
$$

where $S(x, t)$ and $z(x, t)$ are two functions. We insert (8) in (7) and consider different orders of expansion in $\epsilon$. At leading order $\epsilon^{-1}$ the auxiliary function $S$ turns out to be the solution of the Hamilton-Jacobi equation for a classical action

$$
\frac{\partial S}{\partial t}=-H\left(\chi, \phi, \frac{\partial S}{\partial \chi}, \frac{\partial S}{\partial \phi}\right)
$$

The Hamiltonian for the system, $H(x, p)=\frac{p^{2}}{2}+p \cdot f$, is known as the WentzellFreidlin Hamiltonian [13]. The function $S$ evolves along the characteristics of (9) according to $\frac{d S}{d t}=\frac{1}{2}\left(p_{\chi}^{2}+p_{\phi}\right)$. In the limit $\epsilon \rightarrow 0$, the activation process is ruled by the trajectory that minimises the "action" $S$ at the boundary. This is the MPEP, and the action calculated along it is the "activation energy". It is a known result that, in this limit, the MPEP reaches the cycle asymptotically $[16,17]$ and tangentially.

\section{Algorithm}

In this section we describe how DIMS integration scheme $[10,11,18]$ can be extended to the investigation of multidimensional systems such as (2)-(4). The method is applicable to systems characterised by a stable attractor with a sizable basin of attraction, where activation takes place via a large fluctuation.

Consider a generic dynamical system of the form $\dot{x}=f(x)+\xi(t)$; we will focus on transitions from a stable attractor $A_{s}$ toward a final saddle $F_{s}$. The process takes place in three steps: in the vicinity of $A_{s}$ the dynamics of the stochastic system is purely diffusive; then, when the system leaves the vicinity of $A_{s}$ (the length scale is defined by $\sqrt{\epsilon}$ ), the escape becomes essentially ballistic, along the MPEP and, in the vicinity of $F_{s}$, finite noise diffusion dominates. In order to take into account the existence of these three regimes, the integration method is built as follow: the 
system is initially set close to $A_{s}$, the exact equations of motion are integrated using a standard SDE (stochastic differential equation) integrator. This reproduces the diffusive regime in the vicinity of $A_{s}$. We also set a boundary at some distance from the stable attractor: this boundary has nothing to do with the boundary of the basin of attraction, but it delineates where we define the diffusive regimes to change to ballistic. When the boundary is crossed, we switch from standard SDE integration to DIMS integration. Although the final result does not appear to depend on the choice of boundary, the boundary should nevertheless be chosen on some rational basis: we require that the boundary should be fairly easily reached by the dynamical system when using straight SDE integration, but also that this event should not be too probable. In practise, a reasonable boundary could be e.g. a circle centred on the stable attractor, with a radius of a few $\sqrt{\epsilon}$. This choice reproduces the diffusive dynamics in the vicinity of the stable state. When the system hits the boundary, we switch to the DIMS integrator: from the above discussion, we know that the SDE $\dot{x}=f(x)+\xi(t)$ maps onto the Hamiltonian equation, so we switch to the integration of

$$
\dot{x}=f(x)+p(x)+\xi(t)
$$

where $p(x)$ is the momentum conjugated with $x$ as obtained from the MPEP and $\xi(t)$ is the DIMS stochastic component. It is clear that in more than one dimension the probability of being exactly on the MPEP during each integration time step is negligible: but the idea is that we should nevertheless use in the integration of (10) the value of $p(x)$ which "pulls" the escape tube toward the boundary. This is achieved by linearising the Hamilton equations near the MPEP, and using the $p(x)$ that keeps the system on the stable manifold of the MPEP. A step of the scheme is explained in Fig.1. Consider a point $A$ in the coordinate space. The momentum $p(x)$ to be used in the equation (10) is calculated in the following way: first, the closest point to $A$ on the MPEP is located, the extended Hamiltonian system is linearized about this point, and the stable and unstable manifolds are built. The point $A^{*}$ in the extended phase space which has the same $x$ coordinate as $A$, and lies on the stable manifold of the MPEP, is located. The momentum of the point $A^{*}$ is then used as $p(x)$ in (10). The integration scheme is interrupted and reset if the trajectory leaves a small neighbourhood of the MPEP (size of a few $\sqrt{\epsilon}$ ).

\section{Results}

We now use the algorithm of Sec. 3 to describe noise-induced polarization switching in VCSELs. Consider a device described by the set of equations (2)-(4) emitting in the fundamental transverse mode [5], whose polarization may switch between two orthogonal directions due to spontaneous emission. The polarization dynamics can be described using the polarization angle $\phi$ and the ellipticity $\chi$. The DIMS scheme is thus applied in the space of the angles $\phi$ and $\chi$ to investigate transitions from the $\hat{y}$-polarized mode to the limit cycle

The most probable escape path has been located by minimizing the action on the set of Hamiltonian trajectories emanating from the initial stationary point. It is a one-parameter set and the space of parameters is diffeomorph to a circle $S^{1}[14,19]$. The most probable escape path is shown in Fig. 2a. It emanates from the stationary point $\chi=0, \phi=\pi / 2$ and reaches the limit cycle asymptotically. In 


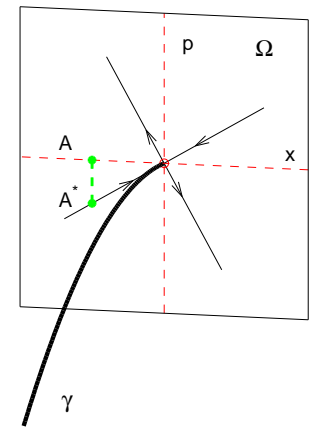

Fig 1. The scheme for one step of the DIMS method, where: $\Omega$ is a cross-section in the extended phase space; $\gamma$ (thick line) is the optimal path predicted by the theory; the thin lines are the stable and unstable eigenspaces (as indicated by the directions of the arrows) of the optimal path. The dashed lines represent respectively the coordinate space $x$ and the momentum space $p$. A is a point in the coordinate space, $A^{*}$ is the corresponding point on the stable manifold of $\gamma$ as required by the integration procedure.

Figs. $2 \mathrm{~b}$ and $2 \mathrm{c}$, the simulations performed with the DIMS method are compared with the theoretical MPEP for two different values of the noise intensity. The 'noisy' trajectories calculated numerically follow very closely the theoretical MPEP; however we are interested in showing how a 'real' trajectory deviates from the theory and leaves the basin of attraction of the lasing mode. In order to show explicitly the shift of the escape point, a collection of 20 numerical trajectories was taken and the point where more than half of the trajectories have left the basin of attraction of the $\hat{y}$-polarized mode is marked. As expected, when the noise intensity increases,
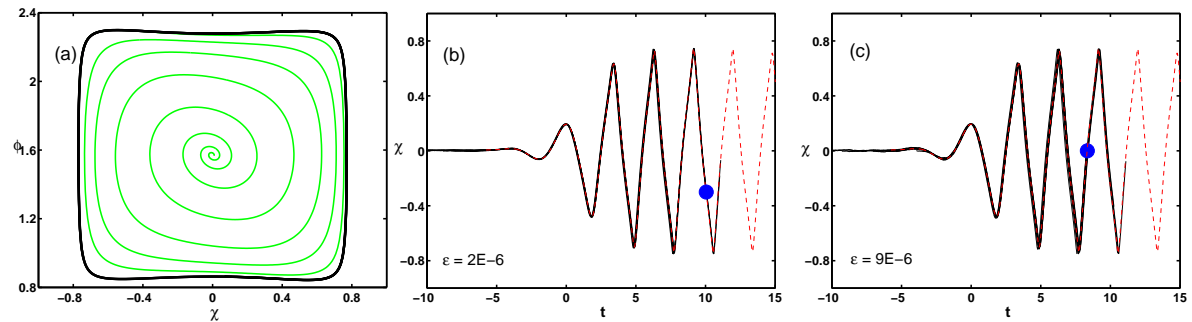

Fig 2. (a) The most probable path for a polarization switch as calculated using the Hamiltonian approach. It emanates from $\chi=0, \phi=\pi / 2$ for $t \rightarrow-\infty$ and reaches the limit cycle tangentially for $t \rightarrow+\infty$. (b),(c) Comparison between theory and simulations for the polarization switches with two different noise intensities: (b) $\epsilon=2 \cdot 10^{-6}$ and (c) $\epsilon=9 \cdot 10^{-6}$. The dashed lines are the theoretical MPEP and the solid lines are the results of the simulation. The full circle marks the point where the probability of a trajectory having left the basin of attraction of the initial lasing mode has exceeded $50 \%$. The dependence of this point on noise intensity is clearly evident.

the simulations follow the MPEP less closely, and they leave the basin of attraction of the initial lasing mode earlier. 


\section{CONCLUSIONS AND DISCUSSION}

In conclusion, we have investigated polarization switching events in VCSELs using an ultra-fast simulation scheme based on dynamical importance sampling of the trajectories. The simulated trajectory is kept close to the MPEP by taking the momentum so as to stay on the stable manifold. By application of the technique we have revealed how a change in noise-intensity affects the switches by modifying the probability of diffusion across the saddle cycle, thus shifting the escape position as shown quantitatively in Figs. 2b and 2c.

These encouraging results on VCSELs suggest that DIMS might provide a way of extending numerical studies to noise intensities that were previously inaccessible, not only for VCSELs but also in many other fields of modern science, e.g. the investigation of ion motion in the pores of cellular membranes [3].

\section{References}

[1] M. I. Dykman, D. G. Luchinsky, R. Mannella and P.V.E. McClintock, N. D. Stein and N. G. Stocks, Stochastic resonance in perspective, Nuovo Cimento D 17 (1995) 661-683.

[2] F. Jülicher, F. Ajdari and J. Prost, Modeling molecular motors, Rev. Mod. Phys. 69 (1997) 1269; M. O. Magnasco, Forced thermal ratchets, Phys. Rev. Lett. 71 (1993) 1477.

[3] R. S. Eisenberg, M. M. Klosek, Z. Schuss, Diffusion as a chemical reaction - Stochastic trajectories between fixed concentrations, J. Chem. Phys. 102 (1995) 1767.

[4] V. I. Mel'nikov, The Kramers problem: fifty years of development, Phys. Rep. 209 (1991) $1-71$.

[5] M. B. Willemsen, M. P. van Exter and J. P. Woerdman, Anatomy of a Polarization Switch of a Vertical-Cavity Semiconductor Laser,

Phys. Rev. Lett. 84 (2000) 4337; M. B. Willemsen, M. P. van Exter and J. P. Woerdman, Correlated fluctuations in the polarisation modes of a vertical-cavity semiconductor laser Phys. Rev. A 604105 (1999);

[6] C. W. Wilmsen, H. Temkin and L. A. Coldren, Vertical-Cavity Surface-Emitting Lasers - Design, Fabrication, Characterization, and Applications (Cambridge, 1999).

[7] H. E. Li, K. Iga, Vertical-Cavity Surface-Emitting Laser Devices (Springer, Berlin, 2003).

[8] K. D. Choquette, R. S. Jr., K. L. Lear, and R. E. Leibenguth, Gain-dependent polarization properties of Vertical-Cavity lasers, IEEE J. Sel. Top. Quantum Electron. 1 (1995) 661-666.

[9] A. Bandrivskyy, S. Beri, D. G. Luchinsky, R. Mannella and P.V.E. McClintock, Fast Monte Carlo Simulations and the Singularities in the Probability Distribution of Nonequilibrium Systems, Phys. Rev. Lett. 90, (2003) 210201.

[10] D. M. Zuckermann and T. B. Woolf, Efficient dynamic importance sampling of rare events in one dimension, Phys. Rev. E 63 (2001) 016702.

[11] S. Beri, R. Mannella, P. V. E. McClintock, Dynamic importance sampling for the escape problem in nonequilibrium systems: observation of shifts in optimal paths, Phys. Rev. Lett. 92 (2004) 020601.

[12] D. Ludwig, Persistence of dynamical systems under random perturbations, SIAM Rev. 17, (1975) 605-640. 
[13] M. Freidlin and A. D. Wentzel, Random Perturbations in Dynamical Systems (Springer, New-York, 1984);

[14] S. Beri, R. Mannella, and P. V. E. McClintock, Dynamics importance sampling for the activation problem in nonequilibrium continuous systems and maps in Noise in Complex Systems and Stochastic Dynamics II, edited by Z. Gingl, J. M. Sancho, L. Schimansky-Geier, and J. Kertesz, Proc. SPIE 5471 (SPIE, Bellingham, WA, 2004) 135-144; S. Beri, R. Mannella, D. G. Luchinsky, and P. V. E. McClintock, Polarization switches in vertical-cavity surface-emitting lasers in Fluctuations and Noise in Photonics and Quantum Optics II, edited by P. Heszler, Proc. SPIE 5468 (SPIE, Bellingham, WA, 2004) 133-141.

[15] A. Bandrivskyy, S. Beri, D. G. Luchinsky, Noise-induced shift of singularities in the pattern of optimal paths, Phys. Lett. A 314 (2003) 386-391.

[16] R. S. Maier and D. L. Stein, A scaling theory of bifurcations in the symmetrical weaknoise escape problem, J. Stat. Phys. 83 (1996) 291-357.

[17] V. N. Smelyanskiy, M. I. Dykman, R. S. Maier, Topological features of large fluctuations to the interior of a limit cycle, Phys. Rev. E 55 (1997) 2369-2391.

[18] T. B. Woolf, Path corrected functionals of stochastic trajectories: towards relative free energy and reaction coordinate calculations, Chem. Phys. Lett. 289 (1998) 433-441.

[19] S. Beri, D. G. Luchinsky, R. Mannella, A. Silchenko, and P. V. E. McClintock, Solution of the boundary value problem for optimal escape in continuous stochastic systems and maps, to be submitted

[20] V. A. Gaisyonok, E. V. Grigorieva and S. A. Kashchenko, Poincaré Mappings for targeting orbits in periodically driven lasers,

Opt.Commun. 124 (1996) 408-417. 